\title{
Theoretical model of the interaction of glycine with hydrogenated amorphous carbon
} (HAC)

Vicente Timón, Óscar Gálvez, Belén Maté, Isabel Tanarro, Víctor J. Herrero and Rafael Escribano.

Instituto de Estructura de la Materia, IEM-CSIC, Serrano 123, 28006 Madrid, Spain. E-mail: vicente.timon@csic.es

\begin{abstract}
A theoretical model of hydrogenated amorphous carbon (HAC) is developed and applied to study the interaction of glycine with HAC surfaces at astronomical temperatures. Two models with different $\mathrm{H}$ content are tried for the HAC surface. The theory is applied at Density Functional Theory (DFT) level, including a semiempirical dispersion correlation potential, d-DFT or Grimme DFT-D2. The level of theory is tested by means of a study of glycine adsorption on a Si (001) surface.
\end{abstract}

Crystalline glycine is also studied in its two stable phases, $\alpha$ and $\beta$, and the metastable $\gamma$ phase. For the adsorption on Si or HAC surfaces, molecular glycine is introduced in the neutral and zwitterionic forms, and the most stable configurations are searched.

All theoretical predictions are checked against experimental observations. HAC films are prepared by plasma enhanced vapor deposition at room temperature. Glycine is deposited at $20 \mathrm{~K}$ within a high vacuum, cold temperature chamber, to simulate astronomical conditions. Adsorption takes place through the acidic group $\mathrm{COO}^{-}$and when several glycine molecules are present, they form $\mathrm{H}$-bond chains among them. Comparison between experiments and predictions suggests that a possible way to improve the theoretical model would require the introduction of aliphatic chains or a polycyclic aromatic core.

The lack of previous models to study the interaction of amino-acids with HAC surfaces provides a motivation for to this work. 


\section{Introduction}

The degree of chemical complexity attainable in different space regions is a subject of intense current investigation. ${ }^{1}$ Special attention has been devoted to the search of organic molecules related to biological compounds. Glycine, the simplest amino acid, has been detected in Solar System objects and its presence is suspected, though not yet firmly established, ${ }^{2}$ in the interstellar medium (ISM) where molecules of comparable complexity have been observed. ${ }^{3}$ The routes toward chemical complexity and thus toward the formation of glycine involve most likely reactions on grain surfaces. ${ }^{1}$ Once formed, glycine would tend to remain on the grains in cold environments, but would desorb from the grains with rising temperature. The equilibrium between adsorption and desorption would be determined by the strength of the interaction between the amino acid and the dust grain surface. The infrared (IR) spectroscopic properties and the survival probabilities of glycine in various astronomical media have been investigated by various groups, see e.g. ${ }^{4-6}$ and references therein.

Other kind of organic compounds of interest in interstellar space are hydrocarbon polymers. In fact a large amount of interstellar carbon is locked into polycyclic aromatic hydrocarbons and carbonaceous dust grains. ${ }^{1,7}$ The composition of these grains is believed to be similar to that of hydrogenated amorphous carbon $(\mathrm{HAC})^{8-10}$, which is a complex disordered form of carbon consisting of diamond-like $\mathrm{sp}^{3}$ and graphite-like $\mathrm{sp}^{2}$ bonded carbon that can exhibit diverse properties depending on its formation conditions. ${ }^{11,12} \mathrm{~A}$ usual way of preparing HAC films in the laboratory is through plasma enhanced chemical vapor deposition (PECVD) of suitable gas precursors. ${ }^{13}$ The IR spectra of HAC films produced through PECVD of small hydrocarbons $\left(\mathrm{CH}_{4}, \mathrm{C}_{2} \mathrm{H}_{2}\right)$ reproduce reasonable well the main absorption bands of interstellar carbonaceous dust. ${ }^{9}$

The interaction of the neutral and ionic forms of glycine (Gly) with the surfaces of metals and oxides has been recently a subject of intense research interest using DFT methods. ${ }^{14-18}$ These studies have allowed a precise characterization of the biomolecule/surface systems at the microscopic level. As far as we know, no such detailed investigations have been carried out for the interactions of glycine with 
analogues of interstellar dust and in particular with HAC. We aim to provide the first model for the study of this effect.

In the present work, we report a thorough DFT study of bulk crystalline glycine, and of the adsorption of this amino acid on HAC and on a Si(001) $2 \times 1$ surface, which is used as a reference. The study is focused on the determination of stable structures, adsorption energies and infrared (IR) spectra for the systems of interest. IR spectra of crystalline $\beta$ glycine and of a Gly/HAC sample were also recorded to provide points of comparison for the predicted spectra.

\section{Experimental}

The experimental set-up consists of a high vacuum cryogenic system coupled to a Vertex 70 FTIR spectrometer. ${ }^{19,20}$ The stainless steel cylindrical chamber, with a residual pressure of $5 \cdot 10^{-8}$ mbar, contains a closed cycle helium cryostat, whose cold finger holds an IR transparent Si window in close thermal contact. The temperature of the Si substrate can be varied between $14 \mathrm{~K}$ and $300 \mathrm{~K}$ with $1 \mathrm{~K}$ accuracy. The IR spectrometer is arranged in normal incidence transmission configuration. A rotatable flange allows the orientation under vacuum of the Si substrate toward different chamber ports. In one position, it faces a sublimation mini-oven of our own design, used previously for glycine ${ }^{5}$, and alanine deposition. ${ }^{21}$ The oven temperature for glycine sublimation was $140 \pm 5 \stackrel{\circ}{ }$. After glycine deposition the Si substrate was turned to another window to record transmission IR spectra. The spectra shown in this work were recorded with a Mercury Cadmium Telluride (MCT) detector, adding between 50 and 300 scans, with $2 \mathrm{~cm}^{-1}$ resolution. The thickness of the glycine samples was estimated by comparison of the absorbance values recorded in the present measurements with literature spectra for samples of known thickness. Specifically we adopted the spectra of ten Kate et al. ${ }^{22}$ for vapor deposited crystalline glycine at room temperature and those of Gerakines et al. ${ }^{23}$ for amorphous glycine at $13 \mathrm{~K}$.

Glycine samples were also deposited at $20 \mathrm{~K}$ on Si substrates previously covered with a HAC layer. The HAC layer was produced by PECVD in an inductively coupled RF discharge reactor described in detail in Maté et al. ${ }^{19}$. The reactor consisted of a pyrex tube of $4 \mathrm{~cm}$ diameter with a 10 turns $\mathrm{Cu}$ coil placed externally around its central part. 
The coil was fed by a $13.56 \mathrm{Mhz}$ RF generator to produce the plasma, which was maintained a constant power of $40 \mathrm{~W}$ during deposition. A mixture of $\mathrm{CH}_{4}(40 \%)$ and $\mathrm{He}$ (60\%), kept at a pressure of $0.3 \mathrm{mbar}$, was used as discharge precursor. The estimated gas residence time in the reactor was $\sim 1 \mathrm{~s}$. For the present experiments, the Si substrate to be covered by the HAC layer was placed $\sim 5 \mathrm{~cm}$ outside the coil in the direction of gas flow. The deposited films, which were found to reproduce the main mid-IR experimental features of interstellar carbonaceous dust, exhibited series of circular interference fringes, which could be used the estimation of their thickness as indicated in Maté et al. 19.

\section{Theoretical models}

\subsection{Theoretical set-up}

All calculations have been carried out using two modules of the Materials Studio package, ${ }^{24}$ the CASTEP code ${ }^{25}$ and the Amorphous Cell (AC) module. With these, amorphous solids of a given composition, density and temperature of generation can be created. CASTEP is used to refine the geometrical structure searching for a minimum in the potential energy surface. At the minimum, the vibrational spectrum is predicted as a list of wavenumber and intensity values for each normal mode. ${ }^{26}$ CASTEP can be applied to amorphous or crystalline solids, metals, and to bulk structures or surfaces.

For this type of calculations the DFT level is usually chosen, as it offers a balanced level of accuracy and computer requirements. However, in our case we are dealing with molecular crystals with van der Waals interactions resulting from dynamical correlations from fluctuating charge distribution, ${ }^{27}$ and it was necessary to add a semi-empirical dispersion correlation potential. We selected either the so-called dispersion corrected DFT method or d-DFT approach, ${ }^{28}$ or the Grimme DFT-D2 approach. ${ }^{29}$ A similar approach was taken in previous studies of the adsorption of glycine on surfaces. ${ }^{14,} 30$ The generalized gradient approximation (GGA) has been employed with two different types of functionals, PBE ${ }^{31}$ and PW91. ${ }^{32}$ Geometry optimization was carried out using the Broyden-Fletcher-Goldfarb-Shanno optimization scheme ${ }^{33}$ with a convergence threshold on atomic forces of $0.05 \mathrm{eV} / \AA ̊ .$. A 5 k-point mesh was adopted ${ }^{34}$ to ensure good convergence for computed structures and energies. 
For the calculations of vibrational properties, the linear response density functional perturbation theory ${ }^{35}$ implemented in the CASTEP code was used, where the phonon frequencies at the gamma point of the Brillouin zone were computed using atomic displacement perturbations. The frequencies presented in this work have not been scaled to correct for anharmonicity and correspond to the harmonic approximation of the force field.

The main points to consider for the comparison between experimental and predicted spectra concern the special characteristics of the theoretical results. First, since no information is available on bandwidths of the calculated spectra, all vibrational modes are depicted using a Lorentzian broadening function of half width at half maximum (HWHM) specified in each case. In particular some observed bands, especially those with $\mathrm{H}$-bonding character in the $2500-2800 \mathrm{~cm}^{-1}$ range, can be very broad. Moreover, theoretical calculations are performed in the harmonic approximation, and therefore overtones or combination bands are not predicted. Although these bands are normally weaker than the fundamentals, they can have a non-negligible contribution to the observed spectra, mainly in the mid-to-high wavenumber zone. Finally, for the spectral assignment, a visualization of the predicted modes in terms of atomic displacements is available with the CASTEP program. However, it is not always easy to assign modes to specific vibrations in solids, as usually several parts of one molecule, or several molecules within the unit cell, are involved in each mode. Use of numerical values from the eigenvectors of the normal modes is not practical either, since often the vibrations are spread over various normal modes with similar eigenvector coefficients. Besides, more than 700 modes can exist in the larger samples with HAC and several glycine molecules (see below), making this task almost meaningless.

\subsection{Bulk glycine modeling.}

As a previous step to glycine adsorption on different surfaces, we have studied in depth the structural and vibrational properties of the phases of crystalline glycine. Structural results are shown in Tables 1 and 2, and spectroscopic results, in Table 3. Initial geometries were taken from crystallographic data, referenced in Table 1. Table 3 presents the spectral assignment and a comparison with previous investigations. We list the 
calculated intensity for each normal mode, a value that can be relevant to estimate band strengths when experimental data are not available.

Room temperature crystalline glycine takes the zwitterionic form (ZG), ${ }^{+} \mathrm{NH}_{3} \mathrm{CH}_{2} \mathrm{COO}^{-}$, for which three phases have been reported. ${ }^{5}$ The unit cell of the $\alpha$-phase contains 4 molecules, whereas there are only two in the $\beta$ - or the metastable $\gamma$-phase. Since all three have comparable densities, the volume of the $\alpha$-structure is nearly double of that of the other two, and the calculated band intensities for the $\alpha$-phase are much larger than those of the corresponding vibrations for the $\beta$-structure. In the gas phase or in low-temperature matrices, glycine can adopt its neutral form, $\mathrm{NH}_{2} \mathrm{CH}_{2} \mathrm{COOH}$. When glycine is deposited from the vapor phase on a low-temperature substrate, a mixture of the neutral and zwitterion forms is produced, with proportions that depend on the temperature of the substrate. The neutral form irreversibly transforms on the ionic form when the temperature of the substrate is increased. Each species is characterized by its own infrared spectrum; the proportion in the mixture can thus be estimated from the relative intensities of some particular bands or spectral regions.

The results in Table 1 show that the optimized structures do not deviate much from the crystallographic parameters. This ensures a good starting point for the calculation of the vibrational properties of these species. We found that the calculated results of GGA-PBE calculated unit cell parameters in $\alpha$ - and $\beta$-polymorphs are in an excellent agreement with the experiments as shown in Table 1.

Table 1 Crystallographic parameters of the $\alpha-, \beta$ - and $\gamma$-glycine structures. Theoretical results correspond to DFT, GGA-PBE level of calculation. All three unit cells have monoclinic symmetry, with $\alpha$ and $\gamma=90$ ․

\begin{tabular}{ccllllc}
\hline System & $a / \AA$ & $b / \AA$ & $c / \AA$ & $\beta / \mathrm{deg}$ & $\mathrm{Vol}^{\circ} \AA^{3}$ & $\rho /\left(\mathrm{g} / \mathrm{cm}^{3}\right)$ \\
\hline$\beta$-Exp* & 5.08 & 6.27 & 5.38 & 113.20 & 158.13 & $1.62^{* *}$ \\
calculated & 5.05 & 6.22 & 5.39 & 112.55 & 156.65 & 1.59 \\
$\alpha$-Exp* & 5.10 & 11.97 & 5.46 & 114.74 & 310.10 & $1.62^{* *}$ \\
calculated & 5.08 & 11.88 & 5.47 & 111.79 & 305.78 & 1.63 \\
$\gamma$-Exp* & 6.93 & 6.93 & 5.43 & 120 & 225.77 & $1.62^{* *}$ \\
calculated & 6.97 & 6.97 & 5.51 & 120 & 231.90 & 1.61 \\
\hline Ref $^{36 * *}$ Ref $^{37}$ & & & & & &
\end{tabular}


Further comparison with experimental results can be made in terms of the main bond lengths and angles of molecular glycine in the $\beta$-phase. They are collected in Table 2 . As far as we know, this phase had never been studied at this level of theory.

Table 2 Structural data for zwitterion glycine in the $\beta$-phase.

\begin{tabular}{lll}
\hline Parameter & $\begin{array}{l}\text { Calculated, } \\
\text { this work }\end{array}$ & Ref. 38 \\
\hline $\mathrm{C}-\mathrm{C} / \AA$ & 1.53 & 1.54 \\
$\mathrm{C}-\mathrm{N} / \AA$ & 1.49 & 1.48 \\
$\mathrm{~N}-\mathrm{H} / \AA$ & 1.03 & \\
$\mathrm{C}-\mathrm{O} / \AA$ & 1.26 & 1.25 \\
$\mathrm{C}-\mathrm{H} / \AA$ & 1.10 & \\
$\mathrm{~N}-\mathrm{C}-\mathrm{C} / \mathrm{deg}$ & 112.45 & 111.80 \\
$\mathrm{C}-\mathrm{C}-\mathrm{O} / \mathrm{deg}$ & 116.46 & 116.66 \\
$\mathrm{O}-\mathrm{C}-\mathrm{O} / \mathrm{deg}$ & 125.25 & 126.39 \\
\hline
\end{tabular}

Table 3 Assignment of the infrared spectra of glycine crystals in the $\alpha$ - and $\beta$-phases. Very weak bands are not listed. The main observed bands of the experimental spectrum of $\beta$ glycine are also included, measured in this work (Fig. 1) and in ref. 38.

\begin{tabular}{|c|c|c|c|c|c|c|}
\hline $\begin{array}{l}\text { Vibrational } \\
\text { mode }^{a}\end{array}$ & $\begin{array}{l}\alpha \text {-glycine } \\
\text { Theor. }\end{array}$ & $\begin{array}{l}\text { Intens. } \\
\mathrm{Km} / \mathrm{mol}\end{array}$ & $\begin{array}{l}\beta \text {-glycine } \\
\text { Theor. }\end{array}$ & $\begin{array}{l}\text { Intens. } \\
\mathrm{Km} / \mathrm{mol}\end{array}$ & $\begin{array}{l}\beta \text {-glycine } \\
\text { Exp. } \\
\text { This work }\end{array}$ & $\begin{array}{l}\beta \beta \text {-glycine } \\
\text { Exp. } \\
\text { (Ref.38) }\end{array}$ \\
\hline$v \mathrm{NH}_{3}$ o.o.p & 3137.7 & 803 & 3224.1 & 99 & 3183.1 & 3180.2 \\
\hline$v \mathrm{NH}_{3}$ i.p. & 3112.4 & 2116 & 3208.3 & 768 & & \\
\hline$v \mathrm{CH}_{2}$ & 2992.5 & 69 & 2980.7 & 17 & & \\
\hline \multirow[t]{3}{*}{$\mathrm{vNH}_{3}, \mathrm{Hbond}$} & 2855.0 & 5918 & 2852.4 & 2914 & 2982.5 & \\
\hline & 2857.3 & 82 & 2845.0 & 175 & & \\
\hline & 2678.8 & 10085 & 2645.7 & 5110 & 2606.4 & \\
\hline \multirow{3}{*}{$\delta \mathrm{NH}_{3}$} & 1611.8 & 281 & 1628.8 & 127 & 1594.9 & 1658.5 \\
\hline & 1563.7 & 1227 & 1571.1 & 669 & & \\
\hline & 1498.0 & 4130 & 1493.4 & 1807 & 1520.7 & 1512.4 \\
\hline \multirow[t]{2}{*}{$\delta \mathrm{CH}_{2}$} & 1418.2 & 105 & 1420.0 & 36 & 1414.6 & 1444.3 \\
\hline & 1374.2 & 1088 & 1373.4 & 689 & & 1410.2 \\
\hline \multirow[t]{2}{*}{$\omega \mathrm{CH}_{2}, \omega \mathrm{NH}_{3}$} & 1308.6 & 496 & 1309.8 & 403 & 1336.5 & 1334.2 \\
\hline & 1302.1 & 526 & & & & \\
\hline \multirow[t]{2}{*}{$\rho \mathrm{NH}_{3}$} & 1147.1 & 128 & 1138.7 & 140 & 1136.9 & 1134.2 \\
\hline & 1111.9 & 150 & 1095.0 & 138 & 1119.5 & 1117.6 \\
\hline$v \mathrm{CN}$ & 1010.7 & 55 & 1018.2 & 24 & 1041.4 & 1040.6 \\
\hline \multirow{2}{*}{$\rho \mathrm{CH}_{2}$} & 906.2 & 96 & 884.5 & 60 & 917.1 & 915.2 \\
\hline & 903.0 & 132 & & & & \\
\hline$v C C$ & 882.8 & 102 & 883.2 & 95 & 893.9 & 893.2 \\
\hline$\delta \mathrm{COO}$ & 701.6 & 193 & 692.5 & 115 & 702.1 & 701.6 \\
\hline$\omega\left(\mathrm{CO}_{2}\right)$ & 577.1 & 32 & 608.1 & 15 & 607.5 & $606^{b}$ \\
\hline
\end{tabular}




\begin{tabular}{lllllll}
\hline$\tau(\mathrm{CN})$ & 499.3 & 316 & 476.61 & 137 & 482.1 & $502^{\mathrm{b}}$ \\
& & & & 519.8 & \\
\end{tabular}

${ }^{a}$ Abbreviations: $v$ : stretching; $\delta$ : bending; $\omega$ : wagging; $\rho$ : rock $\tau$ :torsion; o.o.p.: out of phase; i.p.: in phase

${ }^{\mathrm{b}}$ Ref. 39

Table 3 presents the assignment of the main features of the infrared spectrum of glycine, with calculated values of wavenumber and intensity for the $\alpha$ - and $\beta$-structures. The predicted spectra are compared to the observed spectrum of the $\beta$-form, recorded at room temperature as shown in Fig. 1. As discussed above, the assignments presented in Table 3 are slightly subjective, and might be revised in some cases. For example, in the $1500-1600 \mathrm{~cm}^{-1}$ region, the $\mathrm{NH}_{3}$ bending is the main mode (see also ref. 40 ), but the asymmetric $\mathrm{COO}$ stretching can be appreciated as well in the atomic motions. 

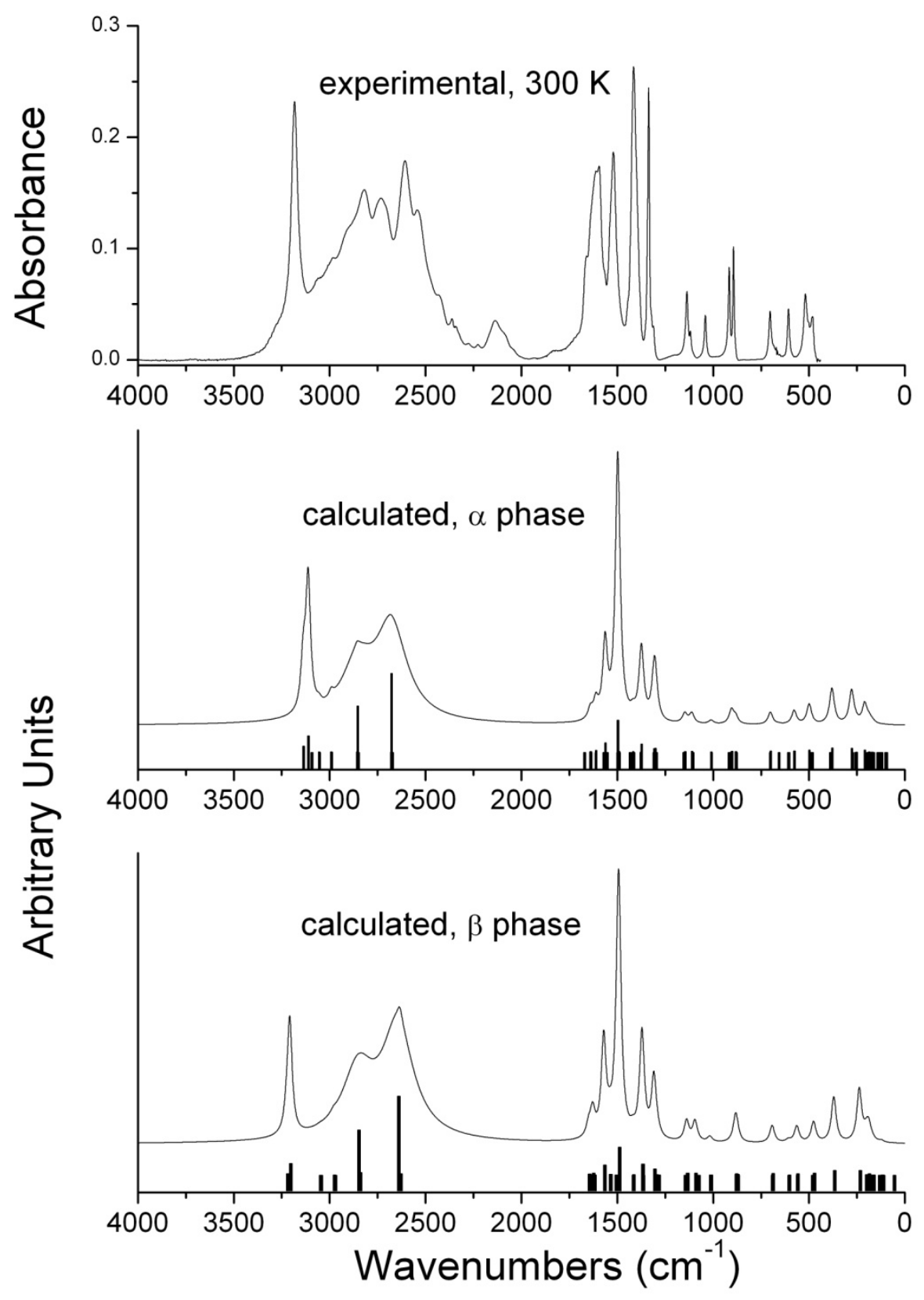

Fig. 1 Infrared spectra of crystalline glycine. Top: experimental spectrum recorded at room temperature, $\beta$-phase. Below, calculated spectra of the $\alpha$ - and $\beta$-phases. The calculated spectra are presented as bars with heights proportional to the calculated intensities, and using Lorentzian functions to broaden the peaks, for a better comparison to the experiment. All vibrations are broadened with HWHM of $15 \mathrm{~cm}^{-1}$, except the H-bond modes, which are broadened with HWHM of $100 \mathrm{~cm}^{-1}$ (see text).

The calculated spectral intensities can be misleading if bandwidths are not taken into account. $\mathrm{H}$-bond vibrations are known to be very strong and wide, and only when they are broadened with a large bandwidth, as in Fig. 1, can a comparison between 
calculated and experimental spectra be meaningful. Even so, our model seems to predict a larger intensity ratio between amino-group vibrations and $\mathrm{CH}_{2}$ or $\mathrm{C}$-involved modes than that observed in the experimental spectrum. Other than that, the main modes are predicted with reasonable wavenumber agreement with the recorded spectrum. The spectra of the $\alpha$ - and $\beta$-crystalline structures look fairly similar, with a small blue-shift of the $\mathrm{vNH}$ modes in the former. The spectra predicted in this work for crystalline $\beta$-glycine give a slightly better agreement with the experimental spectra than those of Stievano et al, $^{38}$ obtained with a similar level of theory.

A peak at $\sim 2150-2200 \mathrm{~cm}^{-1}$ is present in the experimental spectrum, but it is not reproduced in the calculations. This band was observed also by Rosado et $a f^{39}$ who assigned it in the spectrum of the crystalline zwitterionic glycine (Fig. 3 of ref. 39) to a combination mode $\delta_{\text {as }} \mathrm{NH}_{3}+\tau \mathrm{NH}_{3}$. The first one of these modes was assigned to a peak at $1616 \mathrm{~cm}^{-1}$, but the second one was not mentioned in their Table 2. From our calculated values in Table 3, we propose the assignment of this band to a combination $\delta \mathrm{NH}_{3}+\delta \mathrm{COO}$, both of which are strong bands, at $1493.4 \mathrm{~cm}^{-1}$ and $692.5 \mathrm{~cm}^{-1}$, respectively. These second order combination bands are not predicted by our harmonic model, and thus our assignment can only be based on wavenumber coincidence for the combination band and strength of the fundamentals.

\subsection{Silicon $2 \times 1$ (001) surface modeling}

From the theoretical point of view, the construction of a surface model for the study of adsorption of molecules entails two conditions:

(a) A large simulation cell, formed by a single layer with adequate surface area, and a suitable number of slabs to account for all possible interactions.

(b) Relatively limited computational cost, not only for the electronic structure calculation, but also for atomic forces. This last proposition is also important because an accurate force convergence is essential to obtaining a reliable prediction of the infrared spectrum.

These aspects have been taken into account to design the surface models for Si and HAC, prior to the study of glycine adsorption. The $2 \times 1$ (001) surface of silicon has been well 
characterized in previous investigations where silicon was modeled as a substrate for adsorptions of different species. ${ }^{40,41}$ The full procedure used in this work is as follows. We have started by optimizing the Si unit cell, which yielded a lattice constant value of 5.423 $\AA$ and $a \mathrm{~d}_{\mathrm{Si}-S \mathrm{i}}$ value of $2.348 \AA$, very close to the experimental results of $5.43 \AA$ and $2.35 \AA$ respectively. ${ }^{42}$ Next, the $2 \times 1$ (001) surface was selected and optimized, whereby the asymmetric dimer configuration ${ }^{43}$ was formed, with bond distances $d_{\mathrm{Si} \text { Si }}=2.32 \AA$ and a tilt angle of 18 . The slab representing $(2 \times 1)$ coverage used in the calculations reported here contained 7 layers of $\mathrm{Si}$ atoms, terminated by $8 \mathrm{H}$ atoms in a way similar to that described in ref. 46. This slab was doubled in order to get enough surface space to adsorb a few glycine molecules. In the whole, we have dealt with a total of 72 atoms (56 Si and $16 \mathrm{H}$ ). The end $\mathrm{H}$ atoms were included to saturate the $\mathrm{Si}$ dangling bonds in the slab and were initially placed along the bulk Si directions. Then the Si-H distances were optimized using the primitive surface cell with all Si atoms fixed. When calculating reconstructed $\mathrm{Si}$ surfaces, the $\mathrm{H}$ atoms and the bottom two layers of the Si slab were kept fixed while atoms in the rest of the slab were allowed to relax until the forces on individual atoms were less than $0.05 \mathrm{eV} \AA^{-1}$.

\subsection{Hydrogenated amorphous carbon (HAC) surface modeling}

We have followed a similar scheme to the one used by Jacob et al. ${ }^{44}$ to model the HAC surface. In the first step, we have generated two bulk models for HAC particles, called "hard" and "soft" in reference to the amount of hydrogen atoms included in the sample. In both cases, we started by creating a unit cell of cubic symmetry using the Amorphous Cell module of Materials Studio, fixing a temperature of $300 \mathrm{~K}$. For the hard $\mathrm{HAC}$ model, the concentration was $\mathrm{C}_{0.7} \mathrm{H}_{0.3}, \mathrm{H} / \mathrm{C}$ ratio $=0.43$, the density was $2.0 \mathrm{~g} \mathrm{~cm}^{-3}$ and the unit cell side length was $5.24 \AA$. For the soft model, the corresponding values were $\mathrm{C}_{0.5} \mathrm{H}_{0.5}, \mathrm{H} / \mathrm{C}$ ratio $=1$, density, $1.1 \mathrm{~g} \mathrm{~cm}^{-3}$ and unit cell length, $7.23 \AA$. The geometry of the atomic arrangement was then optimized using CASTEP with the parameters specified in Section 3.1, keeping always the volume cell, and therefore the density, fixed. Fig. 2 presents a diagram of the final structure of both models. Convergence to a minimum in the potential energy surface for these models was always a slow process, but for the "soft" system it was also troublesome, as local minima usually prevented the 
achievement of a good relaxation. Moreover, the amount of carbon locked in astrophysical HAC solids has been estimated in an $\mathrm{H} / \mathrm{C}$ ratio of $\approx 0.50,{ }^{45}$ close to the 0.43 value of this work for the "hard" model. Consequently we have chosen the "hard" model system for the rest of the calculations performed in this work.

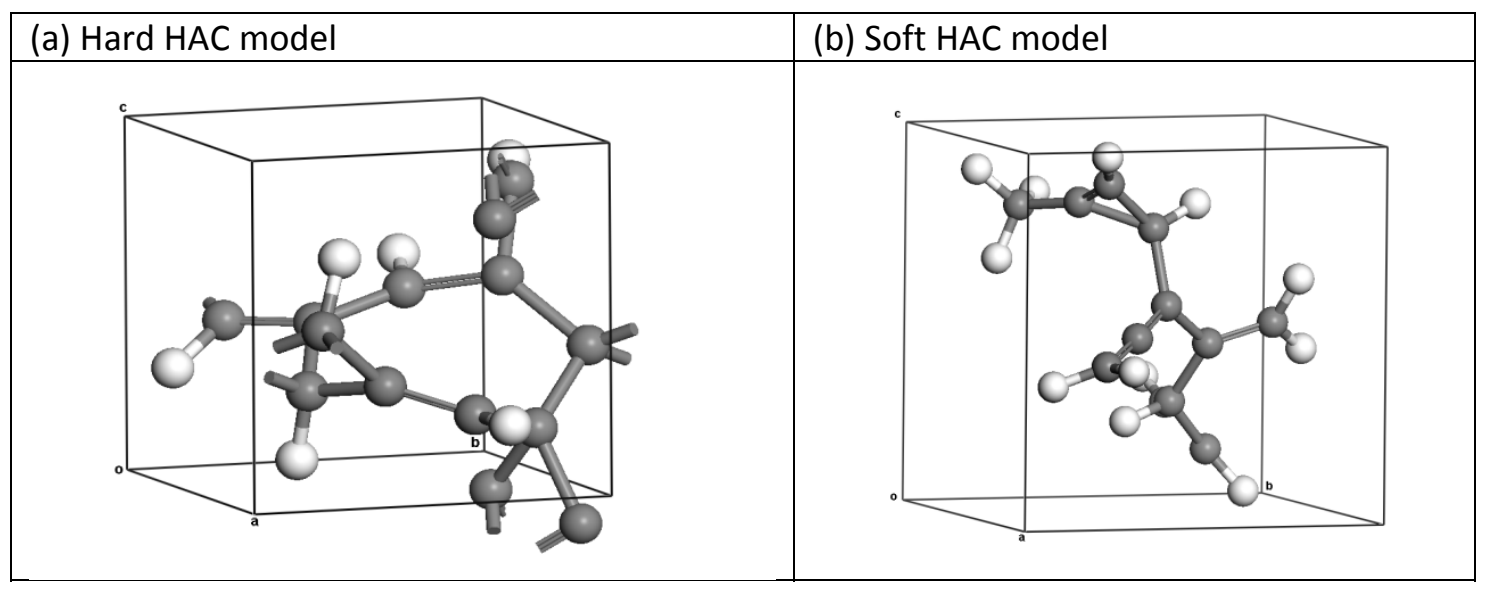

Fig. 2 Optimized geometry of the (a) hard and (b) soft HAC models generated in this work. Hydrogen atoms, white spheres; Carbon atoms, grey spheres.

To study glycine adsorption, one surface of the bulk needs to be selected. Since we have built a cubic cell, in principle we opted by the (001) face. The number of cells necessary to define our system was optimized to ensure a full description of all possible interactions with the adsorbed species. Thus, we chose a $(2 \times 2 \times 2)$ unit cell and created a vacuum of $19 \AA$ along the c-axis to avoid interactions between periodic slabs. The full system then contained $64 \mathrm{H}$ atoms and $144 \mathrm{C}$ atoms. When glycine was added to the upmost surface, the system was allowed to relax until the residual forces on each atom were $0.01 \mathrm{eV} \AA^{-1}$ or smaller, always keeping the volume fixed.

\section{Glycine adsorption on Si and HAC surfaces}

\subsection{Silicon surface}

The adsorption of glycine on a Si surface was studied previously by Qu et al. ${ }^{46}$. These authors reported an energetically more favorable adsorption link through the carboxylic acid part instead of through the amino group of glycine. They discussed 
dissociative adsorption and molecular adsorption. Glycine dissociation on surfaces constitutes an interesting subject on its own, that we intend to tackle in the future. Our preliminary calculations on the dissociation through the acidic part show that this reaction is not produced on Si surfaces, in agreement with Qu et al., ${ }^{46}$ whereas on HAC surfaces the $\mathrm{H}$ atom is initially attached to a $\mathrm{C}$ atom of the surface, but during the relaxation process, it finds its way back to the molecule. Further work needs to be carried out to check and enlarge this study. In the present investigation we deal with molecular adsorption only.

With this fairly simple system, we can concentrate on the details of the adsorption at molecular level. These include, for example, whether glycine adopts the neutral or zwitterionic structure for the surface attachment, whether the presence of multiple glycine molecules affects the system, and if the results of Qu et al. are corroborated.

The structure of neutral glycine (NG) used in this investigation for adsorption purposes has been taken from the electronic ground state calculation of Pacios et $a l^{47}$, in agreement with conformation I of Ramaekers et al. $^{48}$ and Gutowski et al. ${ }^{49}$. The initial structure of the zwitterionic form (ZG) was also taken from Pacios et al. ${ }^{47}$.

Table 4 summarizes our main results of the adsorption of glycine on the Si surface. Energy values listed in this table have been calculated according to standard definitions. ${ }^{50-}$ ${ }^{52}$ The adsorption energy $\Delta \mathrm{E}_{\mathrm{ads}}$ for a specific coverage is the difference between the total energy of the adsorbed system, $E_{\mathrm{T}}$, and the energy associated to the components. These components are the clean substrate, with energy denoted $E_{\text {surface, }}$ and the aggregate of adsorbate glycine molecules, with energy $E_{\text {gly-aggregate: }}$

$$
\Delta E_{\mathrm{ads}}=E_{\mathrm{T}}-\left(E_{\text {surface }}+E_{\text {gly-aggregate }}\right)
$$

This last term includes the individual molecules plus the possible interactions among them. ${ }^{50}$ It is calculated by placing the molecules inside a cell without the adsorbing surface. A negative value of $E_{a d s}$ indicates therefore that the glycine molecules have been bound to the surface.

The procedure employed consists in placing one or more glycine molecules in different arrangements close to the surface and allowing the system to find the energetically most favorable configurations. 
Exploratory calculations have predicted that the most stable alignment is that where the $\mathrm{C}-\mathrm{C}-\mathrm{N}$ plane of glycine is perpendicular to the surface plane. We have thus started with one NG or one ZG either with the carboxylic group or the amino group closer to the Si surface, labeled $\downarrow$ or $\uparrow$, respectively, followed by calculations with 4 and 6 ZG molecules. Table 4 reveals that adsorption takes place in all cases, with binding via the carboxylic group leading to more stable configurations, and with NG adsorption being clearly preferred, as the ionic structures undergo internal redistribution to adopt the neutral form. The highest adsorption energy for a single molecule is $-0.62 \mathrm{eV}$, indicating that a mild adsorbant-adsorbate interaction is taking place. This value is in good agreement with the calculation of Folliet et al. ${ }^{14},-0.57 \mathrm{eV}$, but far from that calculated by Ferraz and Miotto, ${ }^{40}-1.56 \mathrm{eV}$, for a different silicon surface, Si(001)-(2x2). These authors also use DFT theory within a GGA method, but they do not include corrections for van der Waals interactions, as in this work or in other previous calculations. ${ }^{14,30}$ However, the main differences may arise in their optimized Si surface, which appears to be formed by $\mathrm{Si}$ dimers that were not present in our model.

At low coverage our aim was to investigate whether the Si surface is able to stabilize the zwitterionic form of glycine, or if the neutral one prevails, as experimentally observed for low-temperature solid formed by vapour deposition ${ }^{5}$. Our results agree with the observations, i.e. for a single incident glycine molecule the final structure is always the neutral one. When starting with one $Z G$, the binding to the Si surface converges to the neutral conformation II described by Ramaekers et $a l^{48}$, which is slightly less stable than conformation I. With increasing number of initial glycine molecules, the ZG structure becomes more stable, probably because it favors the creation of $\mathrm{H}$-bonds among several molecules. The last structure listed in Table 4 is shown in Fig. 3. One glycine is bound to a $\mathrm{Si}$ atom via the carboxylic $\mathrm{O}$ atom, making part of a network of $\mathrm{H}$-bonds with other molecules, with $\mathrm{H}$-bond distances varying between 1.7 and $2.7 \AA$. The O-Si distance is calculated as $1.78 \AA$, close to the value of Ferraz and Miotto ${ }^{40}$ of $1.84 \AA$. When more glycine molecules are included, 4 or 6 in the last two rows in Table 4 , the calculated adsorption energies grow to values higher than 4 or 6 times the adsorption energy for a single molecule. In view of Fig. 3, this effect can be explained in terms of other minor 
interactions taking place between the surface $\mathrm{Si}$ atoms and other closer glycine molecules, particularly through the ionic amino groups.

Table 4 Energetics and glycine configuration for glycine molecules adsorbed on the $2 \times 1$ (001) Si surface. Fixed volume is $2920.88 \AA^{3}$ for all systems. Super indexes $\uparrow$ and $\downarrow$ refer to structures with one single glycine whose molecular plane is vertically oriented $\mathrm{CCN}$ or NCC respectively with respect to the Si surface.

\begin{tabular}{cccc}
\hline $\begin{array}{c}\text { Initial } \\
\text { configuration }\end{array}$ & $\begin{array}{c}\text { Final } \\
\text { configuration }\end{array}$ & $\mathrm{E}_{\mathrm{T}} / \mathrm{eV}$ & $\mathrm{E}_{\mathrm{ads}} / \mathrm{eV}$ \\
\hline Si surface $\left(\mathrm{Si}_{\mathrm{s}}\right)$ & 0 & -6234.966 & \\
$\mathrm{Si}_{\mathrm{s}}+1 \mathrm{NG}^{\uparrow}$ & $1 \mathrm{NG}^{\uparrow}$ (conf.I) & -7756.241 & -0.45 \\
$\mathrm{Si}_{\mathrm{s}}+1 \mathrm{NG}^{\downarrow}$ & $1 \mathrm{NG}^{\downarrow}$ (conf.I) & -7756.413 & -0.62 \\
$\mathrm{Si}_{\mathrm{s}}+1 \mathrm{ZG}^{\uparrow}$ & $1 \mathrm{NG}^{\uparrow}$ (conf.II) & -7756.231 & -0.44 \\
$\mathrm{Si}_{\mathrm{s}}+1 \mathrm{CG}^{\downarrow}$ & $1 \mathrm{NG}^{\downarrow}$ (conf.II) & -7756.300 & -0.51 \\
$\mathrm{Si}_{\mathrm{s}}+4 \mathrm{ZG}$ & $3 \mathrm{NG}+1 \mathrm{ZG}$ & -12323.288 & -2.62 \\
$\mathrm{Si}_{\mathrm{s}}+6 \mathrm{GG}$ & $1 \mathrm{NG}+5 \mathrm{GG}$ & -15366.264 & -5.06 \\
\hline
\end{tabular}




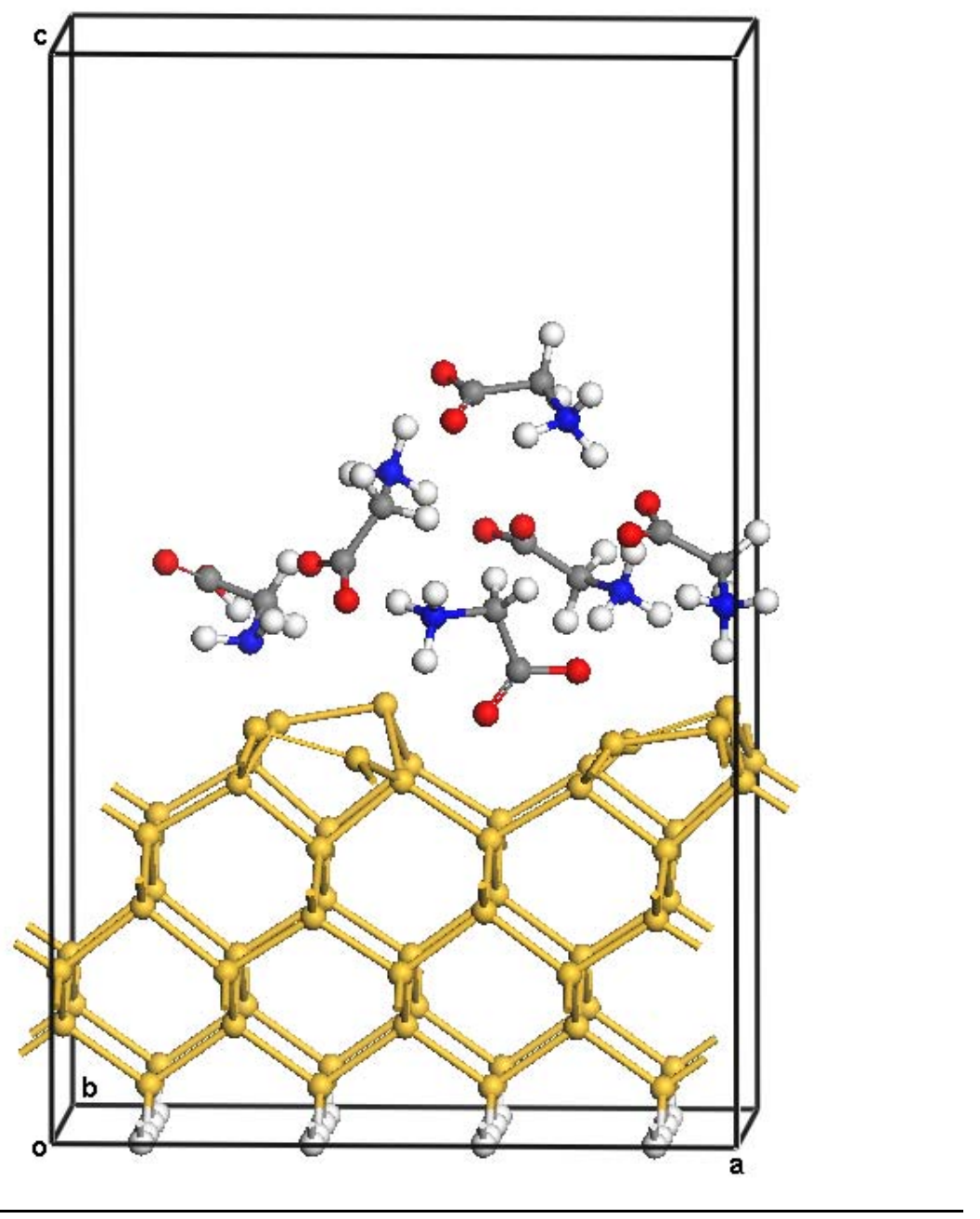

Fig. 3 Optimized structure of the Si 2x1 (001) surface with 6 added glycines. Atoms of hydrogen, carbon, oxygen and nitrogen, white, grey, red and blue spheres, respectively.

\subsection{Amorphous Hydrogenated Carbon (HAC) surface}

A similar procedure to that used for the Si surface was applied to the HAC surface described above. Since this is the most relevant part of this paper, we extended the calculation to cover a larger variety of initial systems. The configuration and adsorption energies for each run are summarized in Table 5.

Table 5 Energetics and glycine configuration for glycine molecules adsorbed on the HAC surface. Fixed volume of $3857 \AA^{3}$ for all systems. 


\begin{tabular}{|c|c|c|c|}
\hline Initial configuration & $\begin{array}{c}\text { Final } \\
\text { configuration }\end{array}$ & $\mathrm{E}_{\mathrm{T}} / \mathrm{eV}$ & $\mathrm{E}_{\mathrm{ads}} / \mathrm{eV}$ \\
\hline HAC-surface $\left(\mathrm{HAC}_{\mathrm{s}}\right)$ & 0 & -23218.826 & \\
\hline NG & - & -1520.986 & \\
\hline ZG & - & -1520.067 & \\
\hline $\mathrm{HAC}_{\mathrm{s}}+1 \mathrm{NG}$ & 1NG & -24739.972 & -0.16 \\
\hline $\mathrm{HAC}_{\mathrm{s}}+1 \mathrm{ZG}$ & 1NG & -24740.110 & -0.30 \\
\hline $\mathrm{HAC}_{\mathrm{s}}+2 \mathrm{NG}$ & $2 N G$ & -26261.039 & -0.05 \\
\hline $\mathrm{HAC}_{\mathrm{s}}+2 \mathrm{ZG}$ & $2 Z G$ & -26262.199 & -0.04 \\
\hline $\mathrm{HAC}_{\mathrm{s}}+4 \mathrm{NG}$ & $3 N G+1 Z G$ & -29305.103 & -0.44 \\
\hline $\mathrm{HAC}_{\mathrm{s}}+6 \mathrm{NG}$ & $6 N G$ & -32347.134 & -0.11 \\
\hline $\mathrm{HAC}_{\mathrm{s}}+5 \mathrm{NG}+1 \mathrm{ZG}$ & $5 N G+1 Z G$ & -32348.794 & -0.05 \\
\hline $\mathrm{HAC}_{\mathrm{s}}+4 \mathrm{NG}+2 \mathrm{ZG}$ & $5 N G+1 Z G$ & -32349.161 & -0.16 \\
\hline $\mathrm{HAC}_{\mathrm{s}}+3 \mathrm{NG}+3 Z \mathrm{G}$ & $5 N G+1 Z G$ & -32347.238 & 1.91 \\
\hline $\mathrm{HAC}_{\mathrm{s}}+2 \mathrm{NG}+4 \mathrm{ZG}$ & $2 N G+4 Z G$ & -32349.547 & 0.32 \\
\hline $\mathrm{HAC}_{\mathrm{s}}+1 \mathrm{NG}+5 \mathrm{ZG}$ & $2 N G+4 Z G$ & -32349.337 & 0.10 \\
\hline $\mathrm{HAC}_{\mathrm{s}}+6 \mathrm{ZG}$ & $1 N G+5 Z G$ & -32349.655 & 0.03 \\
\hline $\mathrm{HAC}_{\mathrm{s}}+12 \mathrm{ZG}$ & $12 Z G$ & $-41677.471^{*}$ & 0.20 \\
\hline $\mathrm{HAC}_{\mathrm{s}}+6 Z \mathrm{ZG}+6 \mathrm{NG}$ & $6 Z G+6 N G$ & $-41673.753^{*}$ & 0.25 \\
\hline $\mathrm{HAC}_{\mathrm{s}}+1 \mathrm{ZG}+11 \mathrm{NG}$ & $1 Z G+11 N G$ & $-41672.395^{*}$ & -0.05 \\
\hline
\end{tabular}

For a single incident glycine molecule, the final configuration is that of the neutral form, attached to the HAC surface via the carboxylic part, similarly to the Si case. The calculated adsorption energy, $-0.30 \mathrm{eV}$, is relatively close to a value calculated for adsorption on graphene, $-0.40 \mathrm{eV} .^{53}$ The shortest distance between glycine and the HAC surface is estimated as $2.4 \AA$. The presence of $\mathrm{H}$ atoms in the HAC surface should favor, in principle, the creation of $\mathrm{H}$-bonds to the carboxylic $\mathrm{O}$ of glycine. However, in comparison to the Si surface case, where the corresponding values for adsorption energy and distance to the surface of a single glycine were $-0.62 \mathrm{eV}$ and $1.78 \AA$, respectively, we can conclude that glycine is more weakly attached to the HAC surface than to the Si surface.

This weak kind of binding interaction may explain why when more glycine molecules are added to the system, the initial NG to ZG ratio is not very much altered, as indicated by the values of Table 5. Fig. 4 shows the lowest energy configuration, where 3 NG and 1 ZG molecules are linked by a network of $\mathrm{H}$-bonds, and also bound to the HAC surface through the carboxylic $\mathrm{O}$ atom of the zwitter molecule, at a distance of $2.66 \AA$. This molecule is not vertically attached to the surface, but tilted, in a position favoring a relaxed structure of the cluster. The overall glycine-HAC interaction is very weak, 
probably as a result of a balance between attractive and repulsive forces between the surface $\mathrm{H}$ atoms, and the carboxylic $\mathrm{O}$ or the amino groups of glycine respectively. Whereas the trend on the Si surface was for glycine molecules to bind individually to the surface, on a HAC surface the glycine molecules tend to group themselves in clusters reducing the adsorption to nearly negligible values. Addition of further molecules does not therefore favor the adsorption.

The configurations of glycine molecules often change along the optimization process. Their motion can be followed with the facilities provided by the CASTEP code. One such video file is deposited with the Supplementary Material.

We tested a bigger system, with 12 glycine molecules. In this case we were forced to employ a different theoretical model to face the large demand on computing resources. We therefore chose ultrasoft pseudopotentials ${ }^{54}$, instead of norm-conserving functions as had been employed for all other calculations, with a cut-off of $350 \mathrm{eV}$. This means that the quoted total energy $E_{T}$ should not be directly compared to the results of the other systems. The adsorption energy $E_{a d s}$ has been calculated as described in formula (1). In these calculations with 12 glycine molecules, we observe two facts: first, the initial configuration ratio ZG/NG is kept; and, second, only when a comparatively large number of NG molecules is included, a favorable system for adsorption, with negative energy, is reached. 


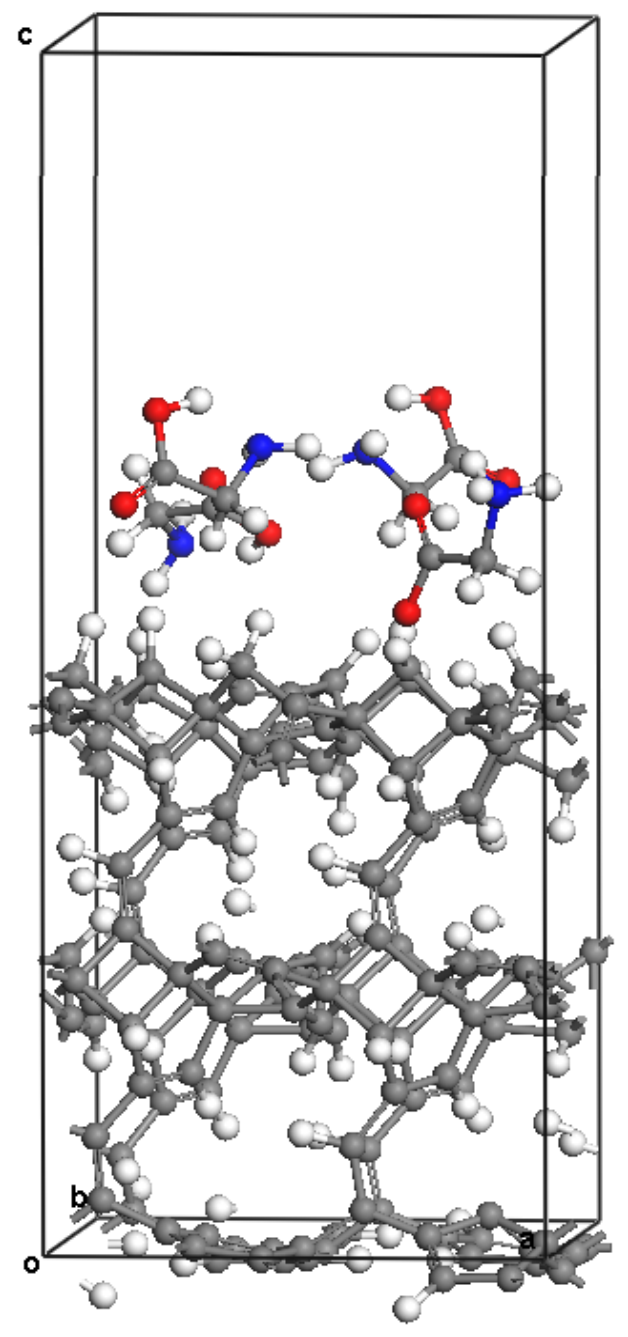

Fig. 4 Representation of the optimized system with the HAC surface and 3NG and 1ZG molecules, the energetically most stable configuration found in this work. Atoms of hydrogen, carbon, oxygen and nitrogen, white, grey, red and blue spheres, respectively.

Fig. 5 presents in the upper panel the recorded spectrum of glycine deposited on a HAC surface at $20 \mathrm{~K}$, obtained as indicated in the experimental section above. At this temperature, the glycine deposit consists of a mixture of NG and ZG molecules ${ }^{5}$ in accordance with the theoretical predictions. The lower panel of this figure displays the predicted spectrum of the sample depicted in Fig. 4, namely the optimized HAC surface with 3 NG and 1 ZG molecules, the energetically most stable configuration found in this work. The estimated thicknesses of the experimental deposits of HAC and glycine are 425 and $140 \mathrm{~nm}$ respectively and correspond roughly to the $1 / 3$ thickness ratio of the 
glycine/HAC model sample used in the theoretical calculations. In the experimental case the IR absorptions correspond essentially to the bulk phases of the two materials, whereas in the calculated spectra a significant fraction of the absorbing species are at the interface between them; however, given the weak interaction between glycine and HAC commented on above, specific interface effects are not expected to be large. A summary of the main peak assignments is given in Table 6. Inspection of Fig. 5 shows that both spectra are characterized by two absorption regions, one between 3300 and $2500 \mathrm{~cm}^{-1}$ with an outstanding feature close $2900 \mathrm{~cm}^{-1}$ and weak absorptions to both sides of it, and another between approximately 2800 and $1000 \mathrm{~cm}^{-1}$ with a series of peaks of comparable magnitude. It must also be born in mind that the predicted spectrum is depicted using a single width value for broadening all modes, irrespective of their nature, which may be quite different in some cases, especially when $\mathrm{H}$-bonds are involved. We have not attempted to use various broadening widths for different modes, as we did in Fig. 1, because of the heavily mixed characteristics of these spectra.

Weak absorptions in the $3300-2500 \mathrm{~cm}^{-1}$ interval, which in the measurements take the form of a continuum, are attributed to $\mathrm{NH}_{3}{ }^{+}, \mathrm{NH}_{2}$ and $\mathrm{CH}_{2}$ stretching vibrations from NG and ZG (cites). The prominent absorption between 3000 and $2800 \mathrm{~cm}^{-1}$, that dominates in the experimental spectrum has three peaks that correspond to $\mathrm{CH}_{3}$ and $\mathrm{CH}_{2}$ stretching vibrations of aliphatic groups in $\mathrm{HAC}^{9,10}$. In the calculated structure there are no methyl groups and consequently only one peak of medium intensity, associated with $\mathrm{CH}_{2}$ stretching vibrations, is observed at $2900 \mathrm{~cm}^{-1}$.

Two comparatively intense bands, characteristic of NG, are observable in the experimental spectrum at 1720 and $1238 \mathrm{~cm}^{-1}$. They are assigned to $\mathrm{C}=\mathrm{O}$ stretching and $\mathrm{COH}$ bending motions respectively. The theoretical predictions do not produce an intense $\mathrm{C}=\mathrm{O}$ band but yield weaker absorptions mixed with $\mathrm{NH}_{\mathrm{x}}$ bendings at 1619 and $1559 \mathrm{~cm}^{-1}$. On the other hand an intense NG absorption is predicted at $1185 \mathrm{~cm}^{-1}$ which most likely corresponds to the observed $1238 \mathrm{~cm}^{-1}$ peak. Other intense peaks assigned to various $\mathrm{NH}_{3}{ }^{+}, \mathrm{NH}_{2}$ and $\mathrm{CH}_{2}$ bending modes and to $\mathrm{COO}^{-}$stretching vibrations of $\mathrm{ZG}$ and $\mathrm{NG}$ molecules are found both theoretically and experimentally between 1670 and $1100 \mathrm{~cm}^{-1}$. In fact, individual assignments in this zone are difficult because the pictorial representations of the vibrations usually show several modes being excited 
simultaneously. The same assertion applies to the low-frequency zone of the spectrum. Simultaneous excitation of various modes takes place preferentially within the same material (either glycine or HAC), but mode mixing between glycine and HAC is rare. The calculated spectrum has also a pronounced peak at $963 \mathrm{~cm}^{-1}$ attributed to $\mathrm{CH}$ bending modes of HAC, which is absent from the measurements. In the experimental spectrum two typical HAC peaks at 1450 and $1375 \mathrm{~cm}^{-1}$ associated with $\mathrm{CH}_{3}$ and $\mathrm{CH}_{2}$ bending vibrations $^{55}$ are also observed, but they have no counterpart in the theoretical predictions. These results further suggest that the calculated HAC structure has too many $\mathrm{CH}$ and too few $\mathrm{CH}_{2}$ groups as compared with the experimental one.

According to Dartois et al. ${ }^{10}$ carbonaceous dust in the diffuse interstellar medium is dominated by an aliphatic/olefinic backbone structure. The structure of the HAC generated in this work is of this type, although the proportion of the $\mathrm{CH}_{\mathrm{x}}$ units (with $\mathrm{x}=1$, 2 or 3 ) is biased towards lower $x$ values in comparison with the observations. Recent publications by other groups ${ }^{7,55}$ indicate however that interstellar carbonaceous dust is likely formed by a polycyclic aromatic core surrounded by short aliphatic chains. These results invite further theoretical work on HAC modeling in order to take into account the new proposed structures.

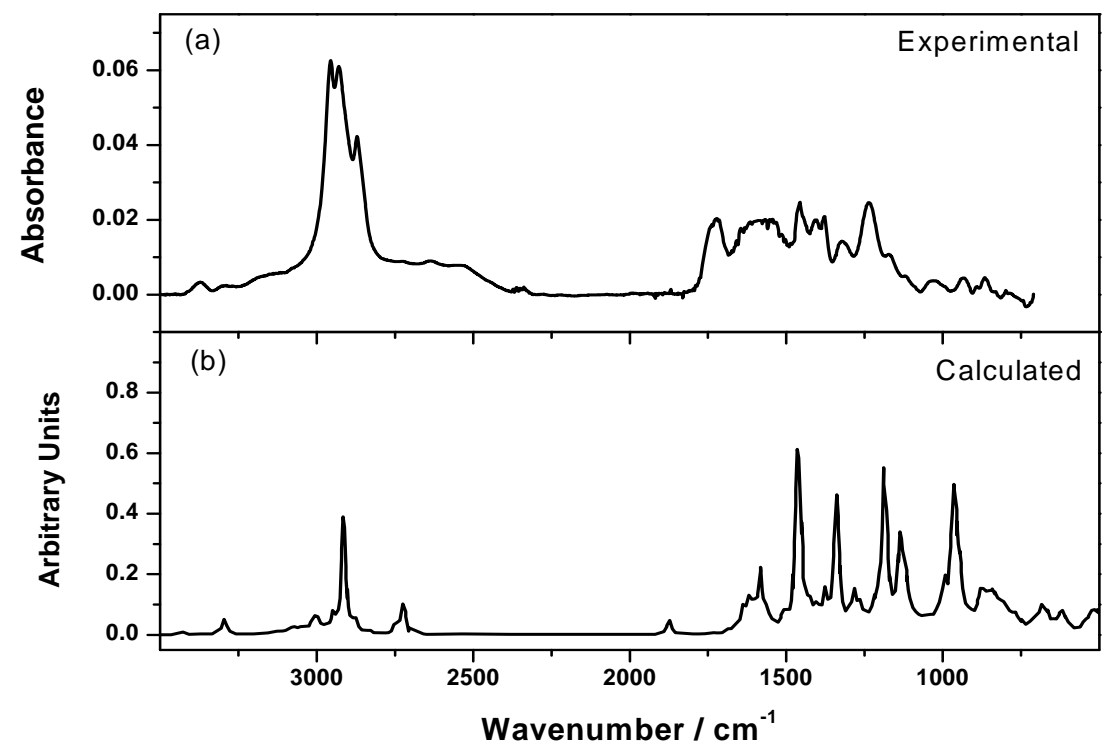

Fig. 5 Infrared spectra of glycine attached to a HAC surface. Above: experimental spectrum recorded at $20 \mathrm{~K}$; below: calculated spectrum for the $\mathrm{HAC}_{\mathrm{s}}+3 \mathrm{NG}+1 \mathrm{ZG}$ model. 
Table 6 Selected unscaled harmonic frequencies $\left(\mathrm{cm}^{-1}\right)$ of 3 Neutral (NG) and 1 Zwitterion (ZG) glycine molecules adsorbed on an amorphous hydrocarbon surface $\left(\mathrm{HAC}_{s}+4 \mathrm{NG}\right)$.

\begin{tabular}{|c|c|c|c|c|}
\hline Vibrational mode & $\operatorname{Exp}^{*}\left(\mathrm{~cm}^{-1}\right)$ & Neutral $\left(\mathrm{cm}^{-1}\right)$ & Zwitterion $\left(\mathrm{cm}^{-1}\right)$ & HAC surface $\left(\mathrm{cm}^{-1}\right)$ \\
\hline$V_{\text {asym }} \mathrm{NH}_{2}$ & $3300-2500$ & 3440 & & \\
\hline$V_{\text {sym }} \mathrm{NH}_{2}$ & $3300-2500$ & 3294 & & \\
\hline $\mathrm{VCH} \mathrm{HAC}_{\mathrm{H}}$ & $3300-2500$ & & & $3118-3000$ \\
\hline$v \mathrm{CH}_{2 \mathrm{HAC}}$ & $3300-2500$ & & & 2984-2910 \\
\hline $\mathrm{V}_{\mathrm{a}} \mathrm{CH}_{2 \mathrm{HAC}}$ & 2928 & & & \\
\hline $\mathrm{V}_{\mathrm{a}} \mathrm{CH}_{3 \mathrm{HAC}}$ & 2956 & & & \\
\hline $\mathrm{V}_{\mathrm{s}} \mathrm{CH}_{3 \mathrm{HAC}}$ & 2871 & & & \\
\hline $\mathrm{vCH}_{2}$ & & 2948 & 3000,2914 & \\
\hline$v_{\text {sym }} \mathrm{NH}_{3}$ & $3300-2500$ & & 2878 & \\
\hline$V_{\text {asym }} \mathrm{NH}_{3}$ & $3300-2500$ & & 2832 & \\
\hline $\mathrm{vOH}$ & & 2722 & & \\
\hline$v C-C$, no $C-H$ & & & & 1873 \\
\hline$v C=C$, no $C-H$ & & & & 1684 \\
\hline $\mathrm{VC}-\mathrm{C}$, with $\mathrm{C}-\mathrm{H}$ & 1720 & & & 1636 \\
\hline$v C=0$ & $1 / \angle 0$ & & & \\
\hline $\mathrm{vaCOO}_{\mathrm{a}}$ & $1680-1490$ & & & \\
\hline$\delta \mathrm{NH}_{2}$ & $1680-1490$ & 1638,1583 & & \\
\hline$\delta \mathrm{NH}_{3}$ & $1680-1490$ & 1606 & 1619,1580 & \\
\hline$v C=C$, with $\mathrm{C}-\mathrm{H}$ & & & & 1600 \\
\hline$\delta \mathrm{NH}, v \mathrm{C}=\mathrm{O}$ & & & 1559 & \\
\hline $\mathrm{Umb} \mathrm{NH}_{3}$ & & & 1464 & \\
\hline$\delta \mathrm{OH}$ & & 1458,1450 & & \\
\hline$\delta \mathrm{CH}_{2}+\delta \mathrm{CH}_{3}$ & 1456 & & & \\
\hline$\delta \mathrm{CH}_{2}$ & 1406 & & & \\
\hline$v$ coo & 1406 & & & \\
\hline$\delta_{\mathrm{s}} \mathrm{CH}_{2}$ & 1375 & & & \\
\hline$\omega \mathrm{CH}_{2}, \omega \mathrm{NH}_{3}$ & 1325 & & 1338 & \\
\hline$\delta \mathrm{COH}+v \mathrm{CO}$ & 1238 & & & \\
\hline$\tau \mathrm{CH}_{2}$ & 1170 & & & \\
\hline$v \mathrm{CN}$ & 1129 & & & \\
\hline$\rho \mathrm{NH}_{2}, \rho_{\mathrm{NH}_{3}}$ & & 1134 & 1134 & \\
\hline$\delta$ or $\omega \mathrm{CH}$ & & & & 965,957 \\
\hline$v \mathrm{CC}+\omega \mathrm{NH}_{2}$ & 934 & & & \\
\hline$\omega \mathrm{CH}_{2}$ & & & 876,872 & \\
\hline$\omega \mathrm{NH}_{2}+v \mathrm{CC}$ & 862 & & & \\
\hline
\end{tabular}


*This work (Fig. 5)

\section{Summary and Conclusions}

Theoretical calculations of the structure and IR spectra of crystalline glycine and of glycine adsorbed on HAC have been performed at the DFT level. The adsorption of glycine on a Si surface has also been studied. The results of the calculations have been compared with spectra of $\beta$ glycine and of gly/HAC samples generated in our laboratory and with literature data whenever available. The main conclusions of the work are listed below.

Our theoretical methodology allows a correct reproduction of the crystalline structure of $\alpha$ and $\beta$ glycine, and of their IR spectra. The calculations include dispersion forces. As far as we know the structure and IR spectrum of $\beta$ glycine had never been calculated at this level of theory.

The characterization of the Si surface is straightforward, but for the hydrogenated amorphous carbon (HAC), different models are possible. We tested two models, with different $\mathrm{H}$ content, and chose the one with lower H proportion ("hard") because it was reported to be closer to the astrophysical observations.

The adsorption of glycine on Si or HAC surfaces is studied by allowing a variable number of glycine molecules to approach the surface, and then by searching for a minimum in the potential energy of the surface+adsorbate systems. Stable configurations are found when some binding is created between glycine and the surface. The binding takes place through fairly weak bonds between the carboxylic part of glycine, rather than the amino part, and the surface atoms.

Rather surprisingly, the adsorption of a single glycine molecule on $\mathrm{Si}$ is slightly stronger than on HAC, with shorter binding distance (1.78 A for Si. 2.40 for HAC) and higher adsorption energy ( $-0.62 \mathrm{eV}$ for $\mathrm{Si}$ and $-0.30 \mathrm{eV}$ for $\mathrm{HAC}$ ). This reveals a quite weak reactivity of the HAC surface for glycine, and probably for other amino acids.

When several glycine molecules approach the surfaces, the most stable configuration usually consists in glycine clusters formed by $\mathrm{H}$-bonding links among them, being attached 
to the surface through the carboxylic part of a glycine molecule in its zwitterionic structure. Although the structure of glycine molecules may vary between the neutral and zwitterionic forms along the adsorption process, the final outcome of NG vs ZG is usually close to the one chosen for the initial configuration.

The IR spectra calculated for the HAC+ glycine model structures reproduce reasonably well most of the experimental absorptions of a glycine layer deposited on HAC. Spectral features specific of the interface are not relevant in the calculated spectrum, in accordance with the weak interaction found between HAC and glycine. The comparison of experimental and measured spectra suggests that the HAC structure should be refined to accommodate $\mathrm{CH}_{3}$ groups and to diminish the proportion of $\mathrm{CH}$ groups.

\section{Acknowledgements}

This research has been carried out with funding from Spanish MINECO, Projects FIS201348087-C2-1-P and CDS2009-00038 and EU project ERC-2013-Syg 610256. O.G. acknowledges the Ramón y Cajal Program and the project CGL2013-48415-C2-1-R. Some calculations were performed at CESGA and TRUENO. We are grateful to M.A. Moreno for technical help and Francisco Colmenero for useful discussions.

\section{References}

1. A. G. G. M. Tielens, Reviews of Modern Physics, 2013, 85, 1021-1081.

2. L. E. Snyder, F. J. Lovas, J. M. Hollis, D. N. Friedel, P. R. Jewell, A. Remijan, V. V. Ilyushin, E. A. Alekseev and S. F. Dyubko, The Astrophysical Journal, 2005, 619, 914.

3. E. Herbst and E. F. van Dishoeck, Annual Review of Astronomy and Astrophysics, 2009, 47, 427-480.

4. P. Ehrenfreund, M. P. Bernstein, J. P. Dworkin, S. A. Sandford and L. J. Allamandola, The Astrophysical Journal Letters, 2001, 550, L95.

5. B. Maté, Y. Rodriguez-Lazcano, O. Galvez, I. Tanarro and R. Escribano, Physical Chemistry Chemical Physics, 2011, 13, 12268-12276.

6. P. A. Gerakines, R. L. Hudson, M. H. Moore and J.-L. Bell, Icarus, 2012, 220, 647-659.

7. J. E. Chiar, A. G. G. M. Tielens, A. J. Adamson and A. Ricca, The Astrophysical Journal, 2013, 770, 78.

8. W. W. Duley and D. A. Williams, Monthly Notices of the Royal Astronomical Society, 1983, 205, 67P-70P.

9. Y. J. Pendleton and L. J. Allamandola, The Astrophysical Journal Supplement Series, 2002, 138, 75. 
10. E. Dartois and G. M. Muñoz-Caro, A\&A, 2007, 476, 1235-1242.

11. J. Robertson, Materials Science and Engineering: R: Reports, 2002, 37, 129-281.

12. W. Jacob and W. Möller, Applied Physics Letters, 1993, 63, 1771-1773.

13. F. J. Gordillo-Vázquez, V. J. Herrero and I. Tanarro, Chemical Vapor Deposition, 2007, 13, 267-279.

14. N. Folliet, C. Gervais, D. Costa, G. Laurent, F. Babonneau, L. Stievano, J.-F. Lambert and F. Tielens, The Journal of Physical Chemistry C, 2013, 117, 4104-4114.

15. I. Lopes, L. Piao, L. Stievano and J.-F. Lambert, The Journal of Physical Chemistry C, 2009, 113, 18163-18172.

16. L.-C. Chen, T. Uchida, H.-C. Chang and M. Osawa, Electrochemistry Communications, 2013, 34, 56-59.

17. D. Costa, P. A. Garrain, B. Diawara and P. Marcus, Langmuir, 2011, 27, 2747-2760.

18. A. P. Sandoval, J. M. Orts, A. Rodes and J. M. Feliu, in Vibrational Spectroscopy at Electrified Interfaces, John Wiley \& Sons, Inc., 2013, DOI: 10.1002/9781118658871.ch7, pp. 239-265.

19. B. Maté, I. Tanarro, M. A. Moreno, M. Jiménez-Redondo, R. Escribano and V. J. Herrero, Faraday Discuss, 2014, 168, 267-285.

20. E. Carrasco, J. M. Castillo, R. Escribano, V. J. Herrero, M. A. Moreno and J. Rodríguez, Review of Scientific Instruments, 2002, 73, 3469-3473.

21. Y. Rodríguez-Lazcano, B. Maté, O. Gálvez, V. J. Herrero, I. Tanarro and R. Escribano, Journal of Quantitative Spectroscopy and Radiative Transfer, 2012, 113, 1266-1275.

22. I. L. ten Kate, J. R. C. Garry, Z. Peeters, R. Quinn, B. Foing and P. Ehrenfreund, Meteoritics \& Planetary Science, , 2005, 40, 1185-1193.

23. P. A. Gerakines and R. L. Hudson, Astrobiology, 2013, 13, 647-655.

24. MaterialsStudio, MaterialsStudio, http://accelrys.com/products/materials-studio/).

25. S. J. Clark, M. D. Segall, C. J. Pickard, P. J. Hasnip, M. I. J. Probert, K. Refson and M. C. Payne, Journal, 2005, 220, 567.

26. K. Refson, P. R. Tulip and S. J. Clark, Physical Review B, 2006, 73, 155114.

27. J. F. Dobson, K. McLennan, A. Rubio, J. Wang, T. Gould, H. M. Le and B. P. Dinte, Australian Journal of Chemistry, 2001, 54, 513-527.

28. X. Wu, M. C. Vargas, S. Nayak, V. Lotrich and G. Scoles, The Journal of Chemical Physics, 2001, 115, 8748-8757.

29. S. Grimme, Journal of Computational Chemistry, 2006, 27, 1787-1799.

30. P. A. Garrain, D. Costa and P. Marcus, The Journal of Physical Chemistry C, 2010, 115, 719727.

31. J. P. Perdew, K. Burke and M. Ernzerhof, Physical Review Letters, 1996, 77, 3865-3868.

32. J. P. Perdew and Y. Wang, Physical Review B, 1992, 45, 13244-13249.

33. V. Vassiliadis and R. Conejeros, in Encyclopedia of Optimization, eds. C. Floudas and P. Pardalos, Springer US, 2001, DOI: 10.1007/0-306-48332-7_48, ch. 48, pp. 222-223.

34. H. J. Monkhorst and J. D. Pack, Physical Review B, 1976, 13, 5188-5192.

35. S. Baroni, S. de Gironcoli, A. Dal Corso and P. Giannozzi, Reviews of Modern Physics, 2001, 73, 515-562.

36. A. Dawson, D. R. Allan, S. A. Belmonte, S. J. Clark, W. I. F. David, P. A. McGregor, S. Parsons, C. R. Pulham and L. Sawyer, Crystal Growth \& Design, 2005, 5, 1415-1427.

37. R. C. Houck, Journal of the American Chemical Society, 1930, 52, 2420-2420.

38. L. Stievano, F. Tielens, I. Lopes, N. Folliet, C. Gervais, D. Costa and J.-F. Lambert, Crystal Growth \& Design, 2010, 10, 3657-3667.

39. M. T. Rosado, M. L. T. S. Duarte and R. Fausto, Vibrational Spectroscopy, 1998, 16, 35-54.

40. A. C. Ferraz and R. Miotto, Brazilian Journal of Physics, 2006, 36, 309-312.

41. D. Shemesh, J. Mullin, M. S. Gordon and R. B. Gerber, Chemical Physics, 2008, 347, 218228.

42. R. W. G. Wyckoff, Crystal Structures 1, 1963. 
43. S. Tang, A. J. Freeman and B. Delley, Physical Review B, 1992, 45, 1776-1783.

44. W. Jacob, Thin Solid Films, 1998, 326, 1-42.

45. G. F. Douglas, W. L. John and N. W. Adolf, The Astrophysical Journal, 1999, 526, 752.

46. Y.-Q. Qu, Y. Wang, J. Li and K.-L. Han, Surface Science, 2004, 569, 12-22.

47. L. F. Pacios, O. Gálvez and P. C. Gómez, The Journal of Physical Chemistry A, 2001, 105, 5232-5241.

48. R. Ramaekers, J. Pajak, B. Lambie and G. Maes, Journal of Chemical Physics, 2004, 120, 4182-4193.

49. M. Gutowski, P. Skurski and J. Simons, Journal of the American Chemical Society, 2000, 122, 10159-10162.

50. M. L. Bocquet, A. M. Rappe and H. L. Dai *, Molecular Physics, 2005, 103, 883-890.

51. S. Sitamraju, J. Xiao, M. J. Janik and C. Song, The Journal of Physical Chemistry C, 2015, DOI: 10.1021/jp510326h.

52. M. Tutuianu, O. R. Inderwildi, W. G. Bessler and J. Warnatz, The Journal of Physical Chemistry $B, 2006,110,17484-17492$.

53. N. Dragneva, W. B. Floriano, D. Stauffer, R. C. Mawhinney, G. Fanchini and O. Rubel, The Journal of Chemical Physics, 2013, 139, -.

54. D. Vanderbilt, Physical Review B, 1990, 41, 7892-7895.

55. M. Steglich, C. Jäger, F. Huisken, M. Friedrich, W. Plass, H. J. Räder, K. Müllen and H. Th, The Astrophysical Journal Supplement Series, 2013, 208, 26. 